\title{
Resistencia a la flexión y módulo elástico de resinas de alta, mediana y baja densidad
}

\section{Flexural strength and elastic module of high, medium and low density resins}

\author{
Sezin $\mathrm{M}^{1}$, Lutri $\mathrm{MP}^{1}$, Mirotti $\mathrm{G}^{1}$, Kraemer $\mathrm{ME}^{1}$, Monserrat $\mathrm{N}^{1}$, Piconi $\mathrm{MC}^{1}$, Caballero $\mathrm{AL}^{1}$, Crohare $\mathrm{L}^{1}$ \\ ${ }^{1}$ Universidad Nacional de Córdoba. Córdoba Facultad de Odontología Cátedra de Operatoria I “A”, Argentina
}

\begin{abstract}
Aim: To determine the flexural strength and elastic modulus of high, medium and low density composite resins. Material and methods: 88 specimens of $25 \mathrm{~mm} \times 2 \mathrm{~mm} \times 2 \mathrm{~mm}$ were divided into 11 groups of 8 according to the resin. Group 1: Filtek Z350 XT, group 2: Rok, group 3: Filtek P60, group 4: Filtek Z250 XT, group 5: Polofil Supra, group 6: Te-Econom Plus, Group 7: Grandio, Group 8: Brilliant New Line, Group 9: Prisma AP.H, group 10: Wave Flow and Group 11: Brilliant Flow. Four samples were preserved for 24 hours and four for 30 days. An universal test machine was used with a head speed of $1 \mathrm{~mm}$ per minute by applying a three points load until fracture. Kruskal Wallis test was applied. Results: Flexural strength 24 hours $(M P a)$ : higher values Filtek Z250 XT (112, $25+/-4.52)$, Filtek P60 (110, $75+/-6.43)$ and Grandio (109, $98+/-9,12)$ and lower Brilliant Flow (49, $00+/-2.27) .30$ days: higher values Grandio (124, $35+/-14.78)$, Filtek P60 (119, $98+/-11.60)$ and lower Rok $(59,50+/-4.94)$ with significant differences (p<0,05). Elastic module 24 hours $(\mathrm{GPa})$ : Higher values Grandio (8.33+/-0.46), Filtek Z250 XT (6.96 +/-0.51), Filtek P60 (6.46 +/-0.29) and lower Brilliant Flow (1.52 +/-0.16). 30 days: higher value Grandio (10.51 +/-0.47) and lower Wave Flow $(2.17+/-0.13)$ with significant differences $(\mathrm{p}<0,05)$. The time influenced significantly increasing values for both tests $(\mathrm{p}<0,05)$. Conclusion: Composite resins showed different behaviors. Time factor influenced increasing values excepting Rok and Filtek Z250 XT which kept its approximately equal values.
\end{abstract}

KEY WORDS: Physical properties, viscoelastic properties, composite resins, flexion resistance, elastic module.

\section{RESUMEN}

Objetivo: determinar la resistencia a la flexión y el módulo elástico de resinas compuestas de alta, mediana y baja densidad. Material y Métodos: 88 especímenes de 25mmx2mmx2mm se dividieron en 11 grupos de 8 según la resina. Grupo 1: Filtek Z350 XT, grupo 2: Rok, grupo 3: Filtek P60, grupo 4: Filtek Z250 XT, grupo 5: Polofil Supra, grupo 6: Te-Econom Plus, grupo 7: Grandio, grupo 8: Brilliant New Line, grupo 9: Prisma AP.H, grupo 10: Wave Flow y grupo 11: Brilliant Flow. Cuatro muestras fueron conservadas por 24 horas y cuatro por 30 días. Se empleó una máquina de ensayo universal con una velocidad de cabezal de $1 \mathrm{~mm}$ por minuto aplicando una carga de tres puntos hasta la fractura. Se aplicó la prueba de Kruskal Wallis. Resultados: Resistencia a la flexión 24 horas (MPa): valores mayores Filtek Z250 XT (112,25+/-4,52), Filtek P60 (110,75+/-6,43), y Grandio $(109,98+/-9,12)$ y más bajo Brilliant Flow (49,00+/-2,27). 30 días: valores más altos Grandio (124,35+/-14,78), Filtek P60 $(119,98+/-11,60)$ y más bajo Rok $(59,50+/-4,94)$ con diferencias significativas $(\mathrm{p}<0,05)$. Módulo Elástico 24 horas $(G P a)$ : valores mayores Grandio $(8,33+/-0,46)$, Filtek Z250 XT (6,96+/-0,51), Filtek P60 (6,46+/-0,29) y más bajo Brilliant Flow $(1,52+/-0,16)$. 30 días: valor más alto Grandio $(10,51+/-0,47)$ y más bajo Wave Flow $(2,17+/-0,13)$ con diferencias significativas $(\mathrm{p}<0,05)$. El factor tiempo influyó significativamente aumentando los valores en ambos ensayos $(\mathrm{p}<0,05)$. Conclusión: las resinas compuestas mostraron comportamientos disímiles. El factor tiempo influyó aumentando los valores a excepción de Rok y Filtek Z250 XT que mantuvieron sus valores aproximadamente iguales.

PALABRAS CLAVES: propiedades físicas, propiedades viscoelásticas, resinas compuestas, resistencia a la flexión, módulo elástico. 


\section{Introducción}

La composición de las resinas compuestas a lo largo de los años ha sido modificada para mejorar su comportamiento clínico y posicionarlo como el material de elección más utilizado al momento de realizar una restauración estética ${ }^{1}$.

Las resinas compuestas han experimentado una evolución significativa, principalmente en su composición inorgánica. El relleno ha sido optimizado, aumentando o disminuyendo la proporción de carga y modificando el tamaño, forma $y$ distribución de las partículas. Estas modificaciones mejoran las propiedades físico-mecánicas y otorgan a las resinas compuestas una optimización en su comportamiento clínico ${ }^{2}$.

La resistencia a la compresión, tensión y flexión, son propiedades que están relacionadas con la composición del material en lo referido al tamaño de las partículas de relleno, proporción de carga inorgánica y composición de la matriz orgánica $^{3}$. Si bien las resinas compuestas son ampliamente estudiadas, existen variaciones en los valores de resistencia a la flexión y módulo elástico en relación al tiempo de almacenamiento en el que se realizan estos ensayos, 24 horas o 30 días ${ }^{4-}$ 5-6-7-8

Existen diferentes tipos de resinas compuestas según la densidad. Las resinas fluidas, consideradas de baja densidad, presentan propiedades físico mecánicas diferentes a las de mediana densidad. Poseen sobre todo mayor elasticidad, lo que les permite acompañar los cambios dimensionales del elemento dentario producto de la flexión ${ }^{1}$.

Las resinas compuestas condensables, debido al aumento en su proporción de carga inorgánica, han logrado mejorar su manipulación, viscosidad y resistencia al desgaste, permitiendo una excelente reproducción de la anátomo-morfología oclusal y proximal de los elementos dentarios ${ }^{4}$.

Los nanocomposites contienen partículas de relleno de tamaño nanométrico sueltas o agrupadas en nanoclusters que proveen mecanismos de refuerzo que otorgan mejoras significativas en las propiedades mecánicas como por ejemplo la resistencia al desgaste ${ }^{9}$.

Debido a la importancia que tienen las propiedades físicas en los resultados clínicos de las restauraciones estéticas y teniendo en cuenta los comportamientos disímiles en las propiedades viscoelásticas de las resinas de diferentes densidades, fue propósito de este trabajo determinar la resistencia a la flexión y el módulo elástico en composites de alta, mediana y baja densidad a diferentes tiempos.

\section{Material y métodos}

Para la realización del presente trabajo se prepararon 88 especímenes llenando un molde rígido de elaboración propia, de $25 \mathrm{~mm} \times 2 \mathrm{~mm} \times 2 \mathrm{~mm}$ con resinas compuestas de alta, mediana y baja densidad. Posteriormente se fotopolimerizaron por 20 segundos de cada lado con una unidad LED (Optilux LED Coltène / Whaledent, Altstätten, Switzerland). Las muestras fueron divididas en 11 grupos de 8 especímenes cada uno; catalogándolos del siguiente modo:

- Grupo 1: Filtek Z350 XT (3M ESPE Dental Products. St. Paul, Minnesota, USA)

- Grupo 2: Rok (SDI Limited, Bayswater Victoria, Australia)

- Grupo 3: Filtek P60 (3M ESPE Dental Products. St. Paul, Minnesota, USA)

- Grupo 4: Filtek Z250 XT (3M ESPE Dental Products. St. Paul, Minnesota, USA)

- Grupo 5: Polofil Supra (VOCO, Guxhaven, Germany)

- Grupo 6: Te-Econom Plus (Ivoclar Vivadent, Schaan, Liechtenstein)

- Grupo 7: Grandio (VOCO, Guxhaven, Germany)

- Grupo 8: Brilliant New Line (Coltène/Whaledent, Altstätten,Switzerland)

- Grupo 9: Prisma AP.H (Densply, Konstanz, Germany)

- Grupo 10: Wave Flow (SDI Limited, Bayswater Victoria, Australia) 
- Grupo 11: Brilliant Flow (Coltène/Whaledent, Altstätten, Switzerland)

A su vez, cuatro muestras de cada grupo fueron conservadas en medio acuoso a $37+/-1^{\circ} \mathrm{C}$ por 24 horas y las cuatro 4 restantes por 30 días del mismo modo. Las pruebas se llevaron a cabo con una máquina de ensayo universal para tracción y compresión cuya velocidad de cabezal fue de $1 \mathrm{~mm}$ por minuto (DIGIMESS, modelo RS-800-5 5KN, China) aplicando una carga de tres puntos con extremos libres hasta la fractura (Fig. 1 A y B).

A.

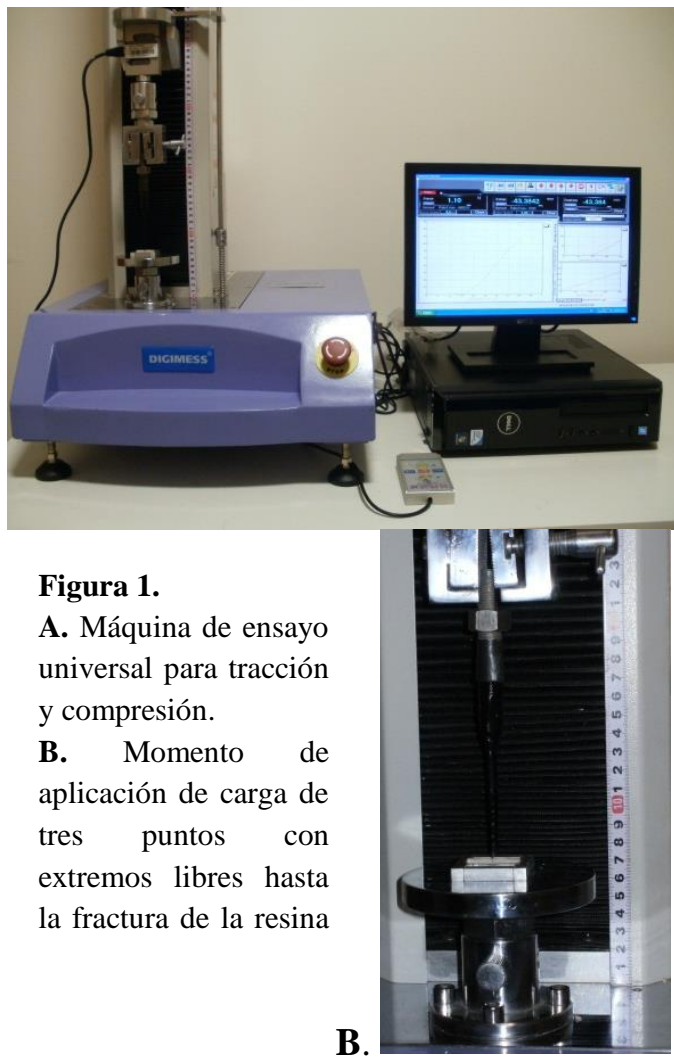

Se consideró resistencia a la flexión como el máximo esfuerzo de una probeta de resina justo antes de que se fracture en un ensayo de flexión. Para obtener los valores resistencia a la flexión en MPa ( $\sigma$ flexión) se aplicó la siguiente fórmula considerando las dimensiones de la probeta:

$$
\frac{3 F L}{2 w h^{2}}
$$

Donde,

$\sigma$ flexión: resistencia a la flexión a tres puntos (MPa)

$\mathrm{F}$ : fuerza de fractura o ruptura $(\mathrm{N}$, Newtons)

L: distancia entre los puntos de apoyo (mm)

W: ancho de la sección de probeta $(\mathrm{mm})$

h: altura de la sección de probeta $(\mathrm{mm})$

En tanto se consideró módulo elástico a la relación entre la deformación de la resina y la fuerza aplicada dentro de un rango de proporcionalidad. El módulo elástico en flexión o módulo de flexión en GPa $(E$ flexión) se calculó en la región elástica de la curva (zona lineal), según la siguiente fórmula:

$\frac{L^{3} F}{4 w h^{3} \delta}$

Donde,

E flexión: módulo de elasticidad en flexión (GPa)

$\delta$ : $\left(\delta_{2}-\delta_{1}\right)$ deflexión o flecha de la probeta en zona elástica $(\mathrm{mm})$

$\mathrm{F}$ : $\left(\mathrm{F}_{2}-\mathrm{F}_{1}\right.$, en zona elástica), fuerza $(\mathrm{N}$, Newtons)

L: distancia entre los puntos de apoyo (mm)

W: ancho de la sección de probeta (mm) h: altura de la sección de probeta (mm)

\section{Análisis estadísticos}

Los datos obtenidos fueron sometidos a la prueba de Kruskal Wallis, prueba no paramétrica, para comparar los valores de resistencia y de módulo de flexión entre los distintos grupos especificados arriba. Se fijó un p-valor $<0.05$ para significación estadística. Para el análisis de los datos se utilizó el software Infostat, versión 2013.

\section{Resultados}

\section{(б) Resistencia a la flexión (MPa)}

En la Tabla 1 se muestran los valores medios y desviación estándar de resistencia a la flexión medidos en MPa a las 24 horas para los diferentes grupos. Se observa que los valores de los grupos 4 Filtek Z250 XT (112,25+/-4,52), 3 Filtek 
P60 (110,75+/-6,43) y 7 Grandio $(109,98+/-9,12) \quad$ fueron mayores significativamente $(\mathrm{p}<0.05)$ en relación a los otros grupos estudiados. Por otra parte, el grupo 11 Brilliant Flow $(49,00+/-2,27)$ fue el que registró el valor significativamente más bajo $(\mathrm{p}<0.05)$.

Tabla 1: Valores medios y desviación estándar (DE) de Resistencia a la flexión expresados en MPa obtenidos a las 24 horas.

\begin{tabular}{l|lllll}
\hline Marca & Medi & DE & $\begin{array}{l}\text { Median } \\
\text { a }\end{array}$ & $\begin{array}{l}\text { Mínim } \\
\text { o }\end{array}$ & $\begin{array}{l}\text { Máxim } \\
\text { o }\end{array}$ \\
\hline Filtek Z250 XT & 112,2 & 4,52 & 112,25 & 106,80 & 117,70 \\
Filtek P60 & 5 & & & & \\
Grandio & 110,7 & 6,43 & 108,75 & 105,90 & 119,60 \\
& 5 & & & & \\
Brilliant & 109,9 & 9,12 & 113,40 & 96,50 & 116,60 \\
Line & 8 & & & & \\
Polofil Supra & 84,58 & 9,91 & 84,15 & 73,50 & 96,50 \\
& & & & & \\
Filtek Z350 XT & 83,83 & 10,2 & 85,70 & 69,90 & 94,00 \\
Te-Econom Plus & 70,48 & 4,92 & 69,10 & 66,30 & 77,40 \\
Wave Flow & 70,48 & 5,38 & 70,70 & 63,70 & 76,80 \\
Prisma AP.H & 68,38 & 5,47 & 66,20 & 64,60 & 76,50 \\
Rok & 64,85 & 6,83 & 64,85 & 57,70 & 72,00 \\
Brilliant Flow & 63,95 & 7,11 & 64,90 & 54,50 & 71,50 \\
\hline & 49,00 & 2,27 & 49,85 & 45,70 & 50,60 \\
\hline
\end{tabular}

En tanto que a los 30 días los valores medios y desviación estándar de resistencia a la flexión medidos en $\mathrm{MPa}$ fueron mayores en el grupo 7 Grandio $(124,35+/-14,78)$ seguido del grupo 3 Filtek P60 $(119,98+/-11,60)$ y el valor más bajo se obtuvo en el grupo 2 Rok $(59,50+/$ $4,94)$ con diferencias significativas en relación a los demás grupos $(\mathrm{p}<0,05)$ (Tabla 2).

Tabla 2: Valores medios y desviación estándar de Resistencia a la flexión expresados en $\mathrm{MPa}$ obtenidos a los 30 días.

\begin{tabular}{|c|c|c|c|c|c|}
\hline Marca & $\begin{array}{l}\text { Medi } \\
\text { a } \\
\end{array}$ & DE & $\begin{array}{l}\text { Median } \\
\text { a }\end{array}$ & $\begin{array}{l}\text { Mínim } \\
\text { o }\end{array}$ & $\begin{array}{l}\text { Máxim } \\
\text { o }\end{array}$ \\
\hline Grandio & $\begin{array}{l}124,3 \\
5\end{array}$ & $\begin{array}{l}14,7 \\
8\end{array}$ & 124,85 & 109,60 & 138,10 \\
\hline Filtek P60 & $\begin{array}{l}119,9 \\
8\end{array}$ & $\begin{array}{l}11,6 \\
0\end{array}$ & 118,85 & 109,50 & 132,70 \\
\hline
\end{tabular}

\begin{tabular}{l|lllll} 
Polofil Supra & 119,4 & 6,09 & 121,40 & 110,60 & 124,20 \\
& 0 & & & & \\
Brilliant New & 117,0 & 6,92 & 118,30 & 107,60 & 124,00 \\
Line & 5 & & & & \\
Filtek Z250 XT & 111,1 & 7,67 & 108,10 & 105,80 & 122,50 \\
& 3 & & & & \\
Te-Econom Plus & 94,67 & 1,27 & 95,30 & 93,20 & 95,50 \\
Filtek Z350 XT & 86,05 & 3,51 & 85,05 & 83,10 & 91,00 \\
Prisma AP.H & 81,78 & 5,54 & 81,50 & 75,30 & 88,80 \\
Wave Flow & 78,78 & 3,60 & 79,50 & 73,90 & 82,20 \\
Brilliant Flow & 69,15 & 5,55 & 68,10 & 63,60 & 76,80 \\
Rok & 59,50 & 4,94 & 61,35 & 52,20 & 63,10 \\
\hline
\end{tabular}

Cuando se evaluó la diferencia entre los tiempos en cada grupo se registró un aumento significativo de los valores de resistencia a la flexión a los 30 días $(p<0,05)$ en casi todos grupos, con excepción de los grupo 4 Filtek Z250 XT y 2 Rok que mantuvieron valores similares en ambos registros de tiempo.

\section{(E) Módulo Elástico en flexión (GPa)}

En la Tabla 3 se muestran los valores medios y desviación estándar del módulo elástico en flexión medidos en $\mathrm{GPa}$ obtenidos a las 24 horas. Se observó que los grupo 7 Grandio $(8,33+/-0,46)$, 4 Filtek Z250 XT $(6,96+/-0,51)$ y 3 Filtek P60 $(6,46+/-0,29)$ fueron mayores significativamente $(\mathrm{p}<0.05)$ en relación a los otros; en tanto el valor significativamente más bajo se obtuvo en el grupo 11 Brilliant Flow $(1,52+/-0,16)$.

Tabla 3. Valores medios y desviación estándar de Módulo Elástico en flexión expresados en GPa obtenido a las 24 horas.

\begin{tabular}{|c|c|c|c|c|c|}
\hline Marca & $\begin{array}{l}\text { Medi } \\
\text { a }\end{array}$ & DE & $\begin{array}{l}\text { Median } \\
\text { a }\end{array}$ & $\begin{array}{l}\text { Mínim } \\
\text { o }\end{array}$ & $\begin{array}{l}\text { Máxim } \\
\text { o }\end{array}$ \\
\hline Grandio & 8,33 & $\begin{array}{l}0,4 \\
6\end{array}$ & 8,55 & 7,64 & 8,58 \\
\hline Filtek Z250 XT & 6,96 & $\begin{array}{l}0,5 \\
1\end{array}$ & 6,90 & 6,44 & 7,62 \\
\hline Filtek P60 & 6,46 & $\begin{array}{l}0,2 \\
9\end{array}$ & 6,38 & 6,23 & 6,85 \\
\hline Polofil Supra & 4,32 & $\begin{array}{l}0,3 \\
8\end{array}$ & 4,20 & 3,99 & 4,87 \\
\hline $\begin{array}{l}\text { Brilliant New } \\
\text { Line }\end{array}$ & 3,31 & $\begin{array}{l}0,2 \\
9\end{array}$ & 3,38 & 2,90 & 3,58 \\
\hline
\end{tabular}




\begin{tabular}{l|cllll} 
Rok & 2,87 & 0,3 & 2,83 & 2,50 & 3,32 \\
& & 5 & & & \\
Te-Econom Plus & 2,80 & 0,2 & 2,75 & 2,56 & 3,13 \\
& & 5 & & & \\
Filtek Z350 XT & 2,34 & 0,1 & 2,34 & 2,15 & 2,54 \\
& & 6 & & & \\
Wave Flow & 1,82 & 0,1 & 1,77 & 1,67 & 2,07 \\
& & 8 & & & \\
Prisma AP.H & 1,75 & 0,1 & 1,75 & 1,65 & 1,86 \\
& & 1 & & & \\
Brilliant Flow & 1,52 & 0,1 & 1,56 & 1,28 & 1,66 \\
& & 6 & & & \\
\hline
\end{tabular}

Los valores evaluados a los 30 días mostraron un cambio significativo del módulo elástico en flexión medidos en $\mathrm{GPa}$, presentando el grupo 7 Grandio $(10,51 \pm 0,47)$ el valor medio más alto y el grupo 10 Wave Flow $(2,17 \pm 0,13)$ el valor medio más bajo $(\mathrm{p}<0,05)$ (Tabla 4$)$.

Tabla 4: Valores medios y desviación estándar de Módulo Elástico en flexión expresados en GPa obtenidos a los 30 días.

\begin{tabular}{|c|c|c|c|c|c|}
\hline Marca & $\begin{array}{l}\text { Medi } \\
\text { a } \\
\end{array}$ & DE & $\begin{array}{l}\text { Median } \\
\text { a } \\
\end{array}$ & $\begin{array}{l}\text { Mínim } \\
\text { o } \\
\end{array}$ & $\begin{array}{l}\text { Máxim } \\
\text { o }\end{array}$ \\
\hline Grandio & 10,51 & $\begin{array}{l}0,4 \\
7\end{array}$ & 10,59 & 9,89 & 10,97 \\
\hline Filtek Z250 XT & 7,06 & $\begin{array}{l}0,5 \\
3\end{array}$ & 7,02 & 6,51 & 7,67 \\
\hline Filtek P60 & 6,78 & $\begin{array}{l}0,3 \\
9\end{array}$ & 6,71 & 6,45 & 7,25 \\
\hline Polofil Supra & 6,07 & $\begin{array}{l}0,4 \\
8\end{array}$ & 6,09 & 5,55 & 6,54 \\
\hline $\begin{array}{l}\text { Brilliant New } \\
\text { Line }\end{array}$ & 5,39 & $\begin{array}{l}0,4 \\
6\end{array}$ & 5,33 & 4,89 & 6,00 \\
\hline Te-Econom Plus & 3,51 & $\begin{array}{l}0,2 \\
0\end{array}$ & 3,59 & 3,28 & 3,66 \\
\hline Filtek Z350 XT & 3,32 & $\begin{array}{l}0,2 \\
2\end{array}$ & 3,34 & 3,07 & 3,51 \\
\hline Prisma AP.H & 3,12 & $\begin{array}{l}0,3 \\
2\end{array}$ & 3,05 & 2,85 & 3,52 \\
\hline Rok & 2,73 & $\begin{array}{l}0,3 \\
4\end{array}$ & 2,81 & 2,30 & 3,02 \\
\hline Brilliant Flow & 2,46 & $\begin{array}{l}0,1 \\
3\end{array}$ & 2,43 & 2,35 & 2,64 \\
\hline Wave Flow & 2,17 & $\begin{array}{l}0,1 \\
3 \\
\end{array}$ & 2,16 & 2,05 & 2,31 \\
\hline
\end{tabular}

La diferencia entre los tiempos en cada grupo registró un aumento significativo de los valores de módulo elástico en flexión a los 30 días $(\mathrm{p}<0,05)$ en casi todos grupos. Se destaca que el grupo 2 Rok y 4 Filtek Z250 XT mantuvieron sus valores aproximadamente iguales.

\section{Discusión}

Las resinas compuestas indicadas en restauraciones del sector posterior o zonas sometidas a cargas oclusales deben presentar valores lo suficientemente altos de resistencia a la flexión y de módulo elástico para soportar deformaciones y evitar fracturas. En tanto que en las preparaciones cavitarias cervicales (clase 5) se recomiendan materiales resinosos que posean propiedades viscoelásticas bajas de manera que acompañen la flexión del elemento dentario ${ }^{1}$. Los efectos e impactos de las propiedades físicas de las resinas compuestas sobre los fenómenos de contracción de polimerización, propiedades de manipulación, durabilidad y resistencia al desgaste en el rendimiento clínico han sido ampliamente investigadas. Los recientes avances tecnológicos centran su desarrollo en la aplicación de la nanotecnología, modificaciones en la carga y tipos de rellenos y mejoras en la morfología, tamaño y distribución de las partículas. Estos cambios influyen de manera directa en las propiedades físicas y en el comportamiento clínico de las resinas compuestas actuales $10,11,12$.

En este trabajo se realizaron ensayos in vitro, porque permiten comparar los valores obtenidos en diferentes institutos y las metodologías estandarizadas de las pruebas. Además, de proporcionar valores físicos que poseen importancia crucial para evaluar la eficacia y seguridad clínica de los materiales 13. Aunque, estos ensayos no siempre permiten una transferencia directa de la eficacia clínica que poseen estos materiales. Así mismo, Ilie $\mathrm{N}$ y col. estudiaron las propiedades físicas de una gran variedad de resinas compuestas mediante pruebas de laboratorio estandarizadas simulando condiciones clínicas. Concluyen que el porcentaje de relleno inorgánico tiene gran influencia sobre la resistencia y módulo de flexión ${ }^{14}$. 
En las pruebas realizadas en nuestro estudio observamos que los valores más altos de resistencia a la flexión, variaron entre 110 a $125 \mathrm{MPa}$, y se registraron en las resinas indicadas para el sector sometido a carga oclusal. La resistencia a la flexión es una propiedad que posee una correlación clínica directa y es de gran valor predictivo para el éxito de un material en la práctica clínica. Las resinas compuestas que presentan valores de resistencia a la flexión de menos de $80 \mathrm{MPa}$ muestran un aumento de fracturas en numerosos estudios clínicos ${ }^{13,15,16}$. La norma ISO determina un valor de resistencia a la flexión de por lo menos 80 MPa como mínimo en zonas sometidas a cargas oclusales y de 90 a $100 \mathrm{MPa}$ para preparaciones cavitarias de clase 2 y 4 , ya que algunos composites exhiben una disminución en la resistencia a la flexión luego de un largo almacenamiento en agua o termociclado. Por lo tanto, las resinas compuestas para el sector posterior deben presentar propiedades viscoelásticas óptimas como por ejemplo alta rigidez para garantizar un comportamiento clínico adecuado y asegurar su longevidad ${ }^{4}$.

Por otra parte, las resinas fluidas estudiadas en este trabajo presentan un valor promedio entre 59 a $80 \mathrm{MPa}$ de resistencia a la flexión. Autores como Attar N y col. afirman que las resinas fluidas, en general, presentan bajas propiedades mecánica y sugieren que estos materiales no deben ser utilizados en las zonas que experimentan una alta carga oclusal. Estos autores observaron valores de resistencia a la flexión en MPa para resina fluida Wave de 66,9 a las 24 horas y 76,3 a los treinta días; similares resultados, en resina Wave, alcanzamos en nuestro estudio, $68,3 \mathrm{MPa}$ a las 24 horas y 78,7 MPa al mes.

Sin embargo, en relación a los valores alcanzados para el ensayo de módulo elástico en $\mathrm{GPa}$ no se encontraron coincidencias entre nuestro estudio $(1,8$ a las 24 horas y 2,1 al mes) y Attar y col. ${ }^{5},(2,8$ a las 24 horas y 4,1 al mes) con igual resina fluida. Estas diferencias podrían deberse a condiciones inherentes al ensayo en nuestro laboratorio, a pesar de que se siguieron estrictamente las recomendaciones de las normas ISO. Sumino N y col. en ensayos de resistencias a la flexión y módulo de elasticidad obtuvieron valores que oscilaron entre 90,5 hasta $135,1 \mathrm{MPa}$ y 4,7 a 7,6 GPa, respectivamente, comparando resinas fluidas y resinas compuestas universales sometidas a ciclos de desgaste localizado ${ }^{17}$.

Las resinas compuestas nanohíbridas utilizadas en nuestro estudio presentaron valores altos de resistencia a la flexión. Ilie y col. demostraron que estas resinas presentan valores elevados de resistencia a la flexión pero bajo módulo elástico, en tanto que las condensables presentan alta resistencia a la flexión ${ }^{14}$.

Kim K y col. afirman que la morfología, el tamaño de las partículas relleno y la proporción de carga inorgánica son factores a considerar cuando se evalúan las propiedades mecánicas y el comportamiento a la fractura de las resinas compuestas. El porcentaje de relleno inorgánico afecta la resistencia y el módulo de flexión como así también la microdureza y la resistencia a la fractura. Los composites que contienen partículas de relleno pre polimerizadas presentan valores significativamente menores de resistencia a la flexión y módulo de flexión respectivamente en comparación con materiales que poseen partículas redondeadas e irregulares ${ }^{18}$. Rodrigues Junior S y col. coinciden en que el contenido de carga interfiriere significativamente en los valores de resistencia a la flexión y módulo de elasticidad de las resinas compuestas ${ }^{19}$.

Además, en otro estudio, Ilie $\mathrm{N}$ y col. analizan las diferencias en las propiedades mecánicas de resinas compuestas convencionales y nanohíbridas. Confirman que estas nuevas tecnologías mejoran los valores alcanzados en los distintos ensayos debido a las diferencias en la cantidad de relleno y el tipo de partículas de los materiales estudiados ${ }^{20}$. También, Curtis A y col. determinan la influencia en los ensayos de resistencia a la flexión que tienen las partículas de relleno nanométricas y los nanoclusters en materiales de base resinosa. Estos autores concluyen que los nanoclusters proporcionaron un mecanismo de refuerzo distinto en comparación con los sistemas resinosos convencionales microhíbridos, microrrellenos o nanohíbridos dando lugar a mejoras significativas en los resultados obtenidos en los ensayos de flexión ${ }^{2}$. 
En nuestros ensayos una de las resinas, Filtex Z250 XT, mostró el valor más alto de resistencia a la flexión $(112,25 \mathrm{MPa})$ en relación a todos los otros tipos de resinas estudiadas. En concordancia con nuestro estudio, Sinval A y col. publican un estudio sobre los valores de resistencia a la flexión en resinas similares (Filtex Z250 y Filtek Supreme) en ensayos de flexión de 3 puntos comparando con pruebas de 4 puntos. Los resultados publicados hacen referencia que ambos materiales obtuvieron comportamientos mecánicos similares, debido al tamaño medio y forma esférica de las partículas de relleno y al porcentaje similar de carga inorgánica, los valores de Filtek Z250 para el ensayo de tres puntos fueron de $127,5 \mathrm{MPa}{ }^{6}$. Investigaciones realizadas por Thomaidis $\mathrm{S}$ y col. obtienen para Filtek Z250 valores de $146 \mathrm{MPa}$ para resistencia a la flexión a las 24 horas y 11,7 GPa en los ensayos de módulo elástico en flexión. Sin embargo la resistencia a la flexión en nuestro estudio, para Filtek Z250XT, mostró valores de $6,96 \mathrm{GPa}$ a las 24h. Está marcada diferencia puede deberse a las propiedades de las resina compuesta que dependen del contenido de carga inorgánica, tipo de materiales de relleno y la eficiencia en el acoplamiento entre las partículas ${ }^{12}$. Y en relación a la metodología de este ensayo, los valores se obtuvieron considerando la fuerza de fractura y el desplazamiento máximo.

Resinas compuestas con alto porcentaje de relleno inorgánico como por ejemplo Grandio ( $87 \%$ de peso y $71,4 \%$ de volumen) tienen altos valores de resistencia a la flexión tanto a las 24 horas como a los 30 días. En un estudio publicado por Sideridou I y col. se establecen valores en MPa de 116,37 a las 24 horas y 116,13 a los 30 días en comparación con los valores obtenidos en este estudio de 109,98 a las 24 horas y 124,35 a los 30 días. Encontramos marcadas diferencias cuando comparamos los valores en los ensayos de módulo elástico en GPa. Estos autores obtuvieron valores de 14,81 a las 24 horas y 14,44 a los 30 días. En tanto que, nuestros resultados fueron de 8,33 a las 24 horas y 10,51 a los 30 días para igual resina compuesta 8 .

Heintze $S$ y col. afirman que los valores de resistencia a la flexión se modifican después del almacenamiento en agua, el valor obtenido a las 24 horas sólo proporciona información limitada. La fiabilidad en los datos se logra cuando se compara el comportamiento del material después de 1 mes en agua ${ }^{13}$.

En este estudio, en relación al factor tiempo, observamos que influye significativamente aumentando los valores de resistencia y módulo de flexión a excepción de las resinas compuestas Rok y Filtek Z250 XT.

Calheiros $\mathrm{F}$ y col. determinan que existen factores que pueden influir en los resultados de las pruebas mecánicas tales como: la temperatura y medio de almacenamiento, dimensiones de la probeta, defectos durante la construcción de las muestras y distribución no homogénea de la luz durante la polimerización, lo que hace dificultoso las comparaciones entre los estudios que utilizan diferentes metodologías. Concluyen que los especímenes más pequeños producen una mayor resistencia a la flexión al igual que el almacenamiento a $37^{\circ} \mathrm{C}$. Además, el almacenamiento con agua llevó a la degradación de los especímenes y por lo tanto, obtuvieron valores más bajos de resistencia a la flexión ${ }^{22}$.

Se requiere de nuevos estudios para poder establecer de qué manera influye del factor tiempo, modo de almacenamiento y las características particulares de cada resina en los valores de resistencia a la flexión y modulo elástico.

\section{Conclusión}

Bajo las condiciones in vitro establecidas en el presente estudio las resinas compuestas de alta, mediana y baja densidad mostraron valores disímiles de resistencia a la flexión y módulo elástico en flexión entre cada una de ellas atribuibles a las características de sus partículas de relleno y proporción del componente inorgánico. El factor tiempo, en ambos ensayos, influyó aumentando en forma significativa los valores obtenidos en MPa a excepción de la resina condensable de alta densidad Rok y microhíbrido de mediana densidad Filtek Z250 XT que mantuvieron sus valores aproximadamente iguales a las 24 horas y 30 días. Nuestros 
resultados confirman que las resinas compuestas con altos valores en propiedades vicoelásticas, es decir de alta o mediana densidad, deben ser aplicadas en zonas sometidas a estrés oclusal, en tanto las resinas compuestas de baja densidad que presentan bajos valores en estas propiedades, se recomiendan para el tercio cervical o zonas libres de contactos masticatorios funcionales.

Todos los autores declaran que no existen conflictos potenciales de interés con respecto a la autoría y/o publicación de este artículo.

All authors declare that there are no potential conflicts of interest with respect to the authorship and / or publication of this article

\section{Referencias}

1. Ruiz JM, Ceballos L, Fuentes MV, Osorio R, Toledano M, García Godoy F. Propiedades mecánicas de resinas compuestas modificadas o no con poliácidos. Avances en Odontoestomatología 2003;19(6): 291-297.

2. Lu H, Lee YK, Oguri M, Powers JM. Properties of a dental resin composite with a spherical inorganic filler. Operative Dentistry 2006;31(6):734-40.

3. Sabbagh J, Ryelandt L, Bachérius L, Biebuyck JJ, Vreven J, Lambrechts P, Leloup G. Characterization of the inorganic fraction of resin composites. J Oral Rehabil. 2004;31(11):1090-101.

4. Papadogiannis $\mathrm{D}$, Tolidis $\mathrm{K}$, Lakes $\mathrm{R}$, Papadogiannis Y. Viscoelastic properties of low-shrinking composite resins compared to packable composite resins. Dent Mater J. 2011;30(3):350-7.

5. Attar N, Tam LE, McComb D. Flow, strength, stiffness and radiopacity of flowable resin composites. J Can Dent Assoc. 2003;69(8):51621.

6. Sinval A, Rodrigues S, Ferracane J, Della Bona A. Flexural strength and Weibull analysis of a microhybrid and a nanofill composite evaluated by 3- and 4-point bending tests. Dent Mater.2008;24(3):426-31.

7. Thomaidis S, Kakaboura A, Mueller W, Zinelis S. Mechanical properties of contemporary composite resins and their interrelations. Dent Mater.2013;29(8): e132-e41.

8. Sideridou I, Karabela M, Vouvoudi E. Physical properties of current dental nanohybrid and nanofill light-cured resin composites. Dent Mater.2011; 27(6): 598-607.
9. Mitra SB, Wu D, Holmes BN. An application of nanotechnology in advanced dental materials. J Am Dent Assoc.2003;134(10):1382-90.

10. Braga RR, Ballester RY, Ferracane JL. Factors involved in the development of polymerization shrinkage stress in resin-composites: a systematic review. Dent Mater. 2005;21(10):962-70.

11. Lin J, Shinya A, Lassila LVJ, Vallittu PK. Composite resin reinforced with pre-tensioned fibers: a three-dimensional finite element study on stress distribution. Odontology. 2013;101(1):29-33.

12. Lin J, Sun M, Zheng Z, Shinya A, Han J, Lin $\mathrm{H}$, Zheng G, Shinya A. Effects of rotating fatigue on the mechanical properties of microhybrid and nanofiller-containing composites. Dent Mater J. 2013;32(3):476-83.

13. Heintze SD, Zimmerli B. Relevance of in vitro tests of adhesive and composite dental materials, a review in 3 parts. Part 1: Approval requirements and standardized testing of composite materials according to ISO specifications. Schweiz Monatsschr Zahnmed 2011; 121:804-816.

14. Ilie N, Hickel R. Investigations on mechanical behaviour of dental composites. Clin Oral Investig. 2009;13(4):427-38.

15. Adabo G, Dos Santos C, Fonseca R, Vaz L. The volumetric fraction of inorganic particles and the flexural strength of composites for posterior teeth. J Dent. 2003;31(5):353-9.

16. Ernst C, Martin M, Stuff S, Willershausen B. Clinical performance of a packable resin composite for posterior teeth after 3 years. Clin Oral Investig. 2001;5(3):148-55.

17. Sumino N, Tsubota K, Takamizawa $T$, Shiratsuchi K, Miyazaki M, Latta MA. Comparison of the wear and flexural characteristics of flowable resin composites for posterior lesions. Acta Odontol Scand. 2013;71(3-4):820-7.

18. Kim K-H, Ong J L, Okuno O. The effect of filler loading and morphology on the mechanical properties of contemporary composites. J Prosthet Dent. 2002;87(6): 642-9.

19. Rodrigues Junior S, Zanchi C, Carvalho R, Demarco F. Flexural strength and modulus of elasticity of different types of resin-based composites. Braz Oral Res. 2007;21(1):16-21.

20. Ilie N, Rencz A, Hickel R. Investigations towards nano-hybrid resin-based composites. Clin Oral Investig. 2013;17(1):185-93.

21. Curtis A, Palin W, Fleming G, Shortall A, Marquis $\mathrm{P}$. The mechanical properties of nanofilled resin-based composites: the impact 
of dry and wet cyclic pre-loading on bi-axial flexure strength. Dent Mater.2009;25(2):18897.

22. Calheiros F, Pfeifer C, Brandão LL, Agra C, Ballester R. Flexural properties of resin composites: influence of specimen dimensions and storage conditions. Dent Mater J. 2013;32(2):228-32
Correspondencia a/Corresponding to: Dr. Mario Sezin: Cátedra de Operatoria I "A". Departamento de Rehabilitación Bucal. Facultad de Odontología. Universidad Nacional de Córdoba Dirección: Haya de la Torre s/n. Ciudad Universitaria. Córdoba. Argentina Correo electrónico/E-mail: mariosezin@hotmail.com 